\title{
Treatment for Spinal Metastasis of Gastrointestinal Stromal Tumors: Two Case Reports
}

\author{
Seung Won Chang, Wonik Cho, Ung-Kyu Chang \\ Department of Neurosurgery, Korea Cancer Center Hospital, Korea Institute of Radiological and Medical Sciences, Seoul, \\ Republic of Korea
}

Corresponding author: Wonik Cho

Department of Neurosurgery, Korea Cancer Center Hospital, Korea Institute of Radiological and Medical Science, 75,

Nowon-ro, Nowon-gu, Seoul 01812, Republic of Korea

Tel: +82-2-970-2322

Fax: +82-2-970-1967

E-mail: 2631@kirams.re.kr

Received: August 9, 2018

Revised: September 2, 2018

Accepted: September 6, 2018
Gastrointestinal stromal tumors (GISTs) are rare, but represent the most common mesenchymal neoplasms of the gastrointestinal tract. The lung and liver are the most frequent sites of distant diseases, but the spinal involvement of metastatic GISTs is very rare. We describe two cases of spinal metastases in patients with advanced GISTs. A 71-year-old female was diagnosed with GIST in 2003 after wedge resection of the stomach. As she had liver metastases, imatinib mesylate was given. Thirteen years later, it was found that she had a sacral metastatic lesion due to buttock pain. Sacral laminectomy and tumor removal surgery were done. Results of the pathologic biopsy showed that she had sacral metastasis from GIST. A 69-year-old male was diagnosed with GIST in 2009 after surgery. Pathologic reviews showed that he had omentum and lymph node metastasis. After surgery, imatinib mesylate was given to the patient as an adjuvant treatment. In 2017, it was found that he had multiple spinal metastatic lesions. A percutaneous biopsy was performed, and pathologic results showed that he had metastasis from GIST. He underwent palliative radiosurgery. Although spine metastases from GISTs are uncommon, metastasis must be considered to the patients who previously had GISTs.

Key Words: Gastrointestinal stromal tumors; Neoplasm metastasis; Radiosurgery; Spinal neoplasms

\section{INTRODUCTION}

Gastrointestinal stromal tumors (GISTs) are rare mesenchymal tumors, the most common form of the gastrointestinal sarcomas accounting for $2 \%$ of all malignant gastrointestinal neoplasms $^{7}$. The diagnosis of GISTs mostly relies on histopathological features and the immunohistochemical (IHC) phenotype. The most important IHC feature is the positivity of the tumor cells for CD117 and CD34. It has been reported in the literature that $90 \%$ to $95 \%, 60 \%$ to $70 \%$, and $30 \%$ to $40 \%$ of GISTs express CD117, CD34, and smooth muscle actin (SMA), respectively. Only $5 \%$ are positive for S-100 protein ${ }^{11)}$. Based on size, mitotic index and anatomic location, GISTs are divided into three groups with low, intermediate, and high-risk. GIST could be differentiated from other mesenchymal tumors such as schwannoma or leiomyoma, according to its distinct IHC characteristics ${ }^{2)}$.

Most of the GISTs have the potential to become malignant, but, a subset of these lesions which are particularly small and asymptomatic are benign in nature ${ }^{14)}$. The incidence of GIST is in the range of 20 to 40 cases per million. Over $90 \%$ of GISTs occur in adults over 40 years old, in a median age of 63 and rarely in children in the second decade $(<1 \%)^{9)}$. Most GISTs are benign; malignant tumors account for $20 \%$ to $30 \%$ of cases.
Its most common anatomic sites of origin are the stomach (60\%$70 \%$ ), small intestine (20\%-30\%), colon and rectum (5\%), abdominal cavity, peritoneum and omentum $(5 \%)$, esophagus $(<5 \%)$ and the retroperitoneal space $(<3 \%)$. Patients have different symptoms, such as abdominal pain and swelling, weakness, and anemia ${ }^{4)}$.

Tumor resection is the treatment of choice for localized disease. The risk of recurrence is identified by evaluating the mitotic index, dimension and site of the tumor ${ }^{2,7)}$. Selective tyrosine kinase inhibitors (imatinib, sunitinib) are the standard therapy for metastatic or unresectable GISTs"). GISTs usually metastasize to the liver and peritoneum. Rare sites of metastasis are lymphnodes, lung and subcutaneous tissue, and intracranial localizations have only been described in a case report ${ }^{3}$. Bone metastases are also uncommon, and GISTs arise very rarely in the spine $^{7)}$.

We describe our treatment experiences of 2 cases of spinal metastases in patients with advanced GISTs.

\section{CASE REPORT}

\section{Case 1}

A 71-year-old female patient was examined due to abdominal 
discomfort developed. The examination and abdominal computed tomography (CT) scans showed a $10 \mathrm{~cm}$ heterogeneous mass with central necrosis and gastric ulcer at the greater curvature of the stomach. Wedge resection of the stomach and splenectomy were performed in March 2003. Results of histopathological examination showed hemorrhage and necrosis, frequent mitosis, and no metastasis in lymph nodes. According to the immunochemical analysis, C-kit and S-100 were positive, and both SMA and calponin were negative. The Ki-67 index was $20 \%$. Tumor diameter was $17.5 \times 12 \mathrm{~cm}$. In February 2004, the patient was examined due to abdominal discomfort redeveloped. Abdominal CT scans showed multiple low attenuating nodules in the liver. Since then, imatinib mesylate $400 \mathrm{mg} /$ day was given to the patient as an adjuvant treatment. The patient had complained of right buttock pain, right leg radiating pain, since February 2016. Magnetic resonance imaging (MRI) scans showed osteolytic bone destruction in the right sacrum. Increased size of soft tissue mass in right piriformis muscle and gluteus muscle across sciatic notch was observed (Fig. 1A). Furthermore, a positron emission tomography (PET)-CT scan was performed on the patient. The results showed increased activities in the right piriformis muscle, right gluteus muscles, and right sacrum (standardized uptake value [SUVmax], 8.7) (Fig. 1B). Sacral laminectomy and tumor removal were done in May 2016 (Fig. 1C). Well marginated mass lesions involving piriformis muscle, glu- teus muscle and sacral body were observed. Pain complaint significantly decreased after surgery. Results of histological examination showed spinal metastasis from GIST (Fig. 2). After the surgery, the patient underwent radiosurgery to residual tumor in the sacrum. 20 gray (Gy) radiation dose in single fraction and $36 \mathrm{~Gy}$ radiation dose divided into 4 fractions were given to the residual tumor. The patient switched chemotherapy imatinib to sunitinib and is showing no sign of recurrence.

\section{Case 2}

A 69-year-old male patient was examined due to abdominal discomfort. The examination and abdominal CT scans showed the presence of a about $11 \mathrm{~cm}$ sized a low attenuating mass in the peri-gastric area. Wedge resection of the fundus and distal pancreatectomy and omentectomy were performed in April 2009. According to the immunochemical analysis, C-kit, and CD34 were diffuse positive, both S-100 and SMA were negative. The Ki- 67 index was $20 \%$. Tumor diameter was $8.5 \times 8 \mathrm{~cm}$, and mucosal invasion was positive and resection margin was clear. Omentum was reported to have metastatic tumor involvement and lymph nodes metastasis. The pathological examination showed high-risk GIST of the stomach and omental metastasis, and lymph nodes metastasis. After the surgery, imatinib mesylate $400 \mathrm{mg}$ /day was given to the patient as an adjuvant treat-
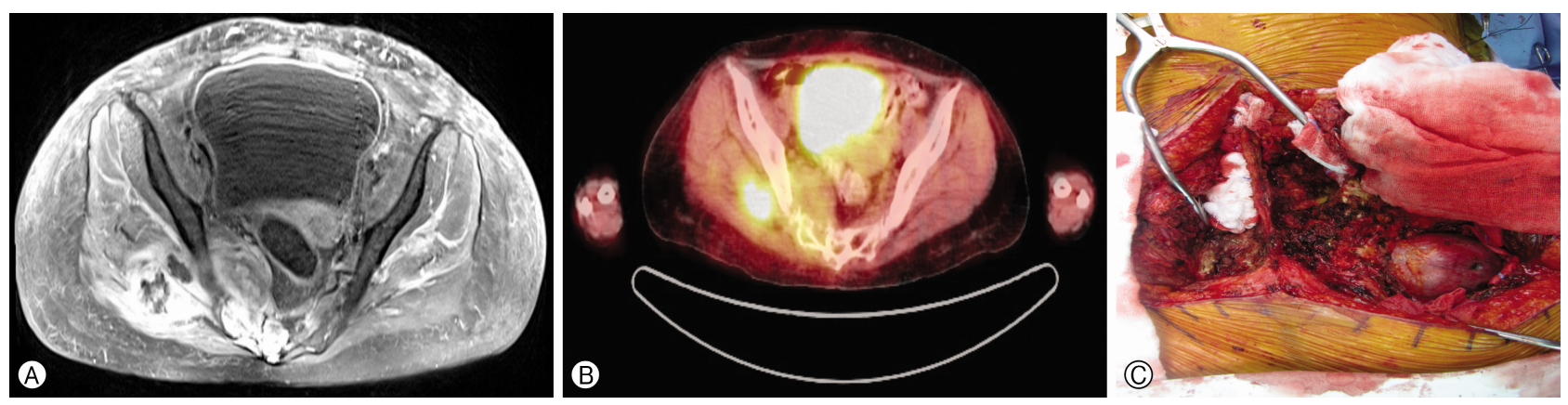

Fig. 1. Images of case 1 patient. (A) Preoperative $\mathrm{T} 1$ enhanced magnetic resonance image of patient before the surgery enhancing mass in the right sacrum, right piriformis muscle, and right gluteus muscle is shown. (B) Preoperative positron emission tomography-computed tomography image of patient before the surgery. Hypermetabolic mass lesion involving right piriformis muscle, right gluteus muscle, and right sacrum is shown. (C) Surgical image of patient. Well-marginated mass lesion involving sacral body and peri-sacral area.
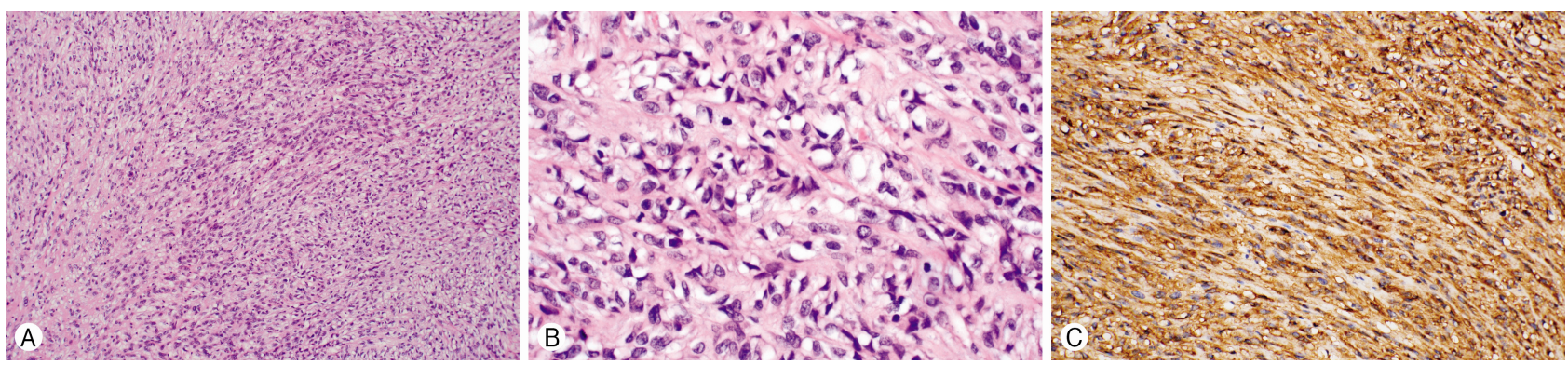

Fig. 2. Histopathologic image of case 1 patient. (A) Hematoxylin and eosin stain $(H \& E), \times 100$ magnification. Tumor cells forming fascicle. Tumor cells have an ovoid shape and have granular to clear chromatin. Most of tumor cells have no nucleoli. (B) $H \& E$ stain $\times 400$. As many as 4 mitoses can be seen in 1 high power field. (C) Immunohistochemistry image showing C-kit $(+)$. 

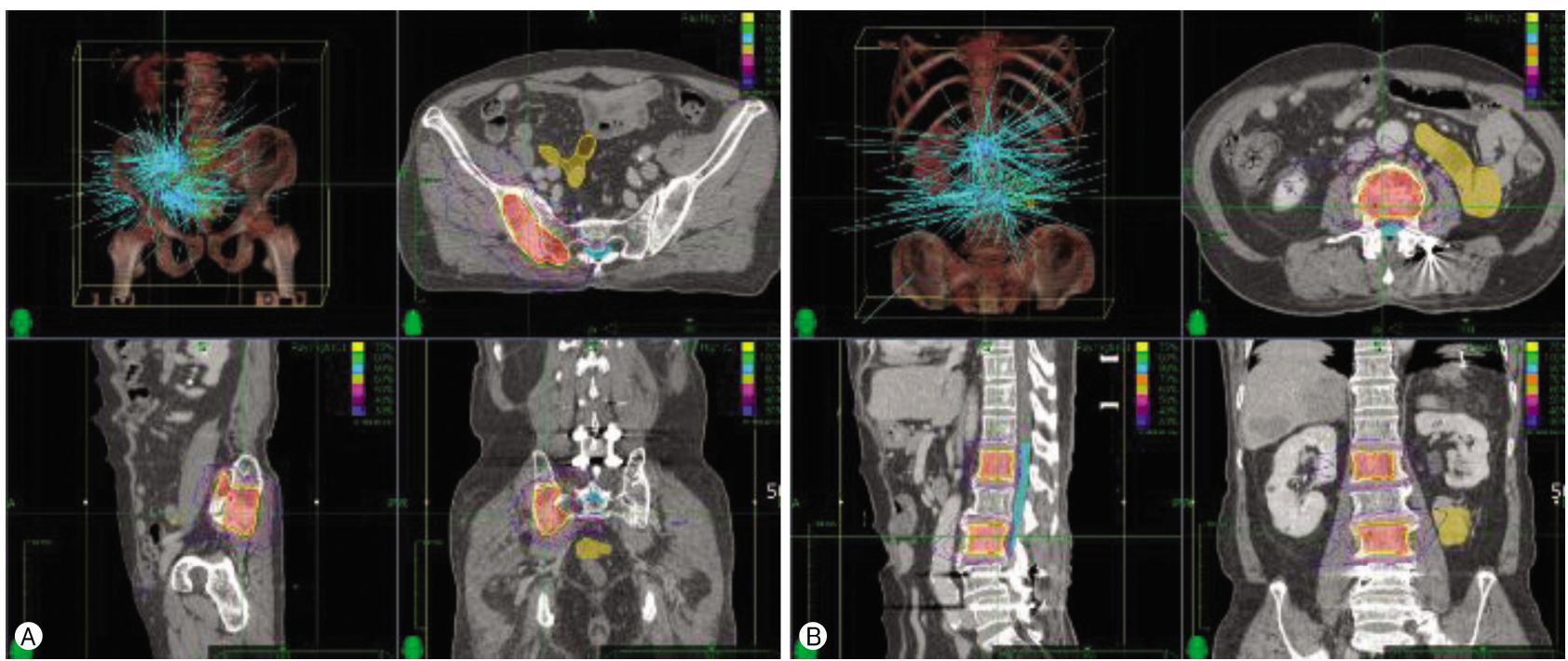

Fig. 3. The case 2 patient palliative stereotactic radiosurgery. Total $36 \mathrm{~Gy}$ of radiation dose was given, divided 4 fractions on the spinal metastasis. (A) Radiosurgery plan for the iliac bone and pelvis. (B) Radiosurgery plan for L1, L3 spine.

ment. In March 2014, the patient was examined due to abdominal discomfort redeveloped. Abdominal CT scans showed mass recurrence in the retroperitoneal space. Metastasectomy was done in May 2014. The patient had complained of right buttock pain and right thigh pain from May 2017. MRI showed osteolytic bone destruction in right sacrum, pelvis and L1, L3 vertebral bodies. We performed a percutaneous needle biopsy at L3 vertebral body by kyphoplastic needle and results of pathological analysis showed spinal metastasis arising from GISTs. Since the patient had multiple metastatic lesions, it was considered impossible to remove all the lesions. Thus, we decided to perform palliative stereotactic radiosurgery (SRS). Total $36 \mathrm{~Gy}$ of radiation dose was given, divided 4 fractions on the spinal metastasis (Fig. 3). Pain complaints significantly decreased after the radiotherapy. After the radiosurgery, the lesions and bone scans showed decreased activities in L1 and L3 spine. In addition, the lesions in the sacrum showed no significant change. However, newly developed and increased uptake in PET scan was observed in the bilateral femur. The patient continued to undergo chemotherapy until follow-up was lost in October, 2016.

\section{DISCUSSION}

GISTs are the most common mesenchymal tumors of the gastrointestinal tract with an incidence of 10 to 20 cases per million of which almost one third are deemed malignant. The usual age at presentation is around 60. GIST may present anywhere in the gastrointestinal tract, omentum, or mesentery. The most common sites are the stomach (60\%), small intestine (15\%), colon and rectum (5\%), other abdominal organs including mesentery, and omentum $(5 \%)^{15)}$. Metastasis is characterized by the malignant behaviors of the GIST. GISTs most frequently make metastasis to the liver and peritoneum, whereas spine and lung metastases are uncommon sites ${ }^{3}$.

Surgical removal of the tumor is currently considered to be the first choice for treatment of GIST, regardless of the presence of distant metastases ${ }^{2,4)}$. Local recurrence and distant metastases have been reported to develop in more than 30\% of patients with high-risk GIST despite apparently complete resections. Most recurrences or metastases occurring after resection with a R0 margin of high-risk GISTs appear within 3 years after primary surgery. An adjuvant therapy with imatinib mesylate, a specific molecular inhibitor, is beneficial after the primary GISTs are resected. Treatment for malignant GISTs using imatinib mesylate can be given at first in the treatment of advanced, recurrent, unresectable or metastatic GISTs. Once an advanced GIST (metastatic, unresectable, or recurrent) is diagnosed, imatinib should be immediately prescribed; regardless of the presence of symptoms and even if the tumor is completely resected grossly and histologically ${ }^{10,13)}$. Three years of adjuvant treatment with imatinib mesylate has been shown to improve recurrence-free survival and overall survival rates in patients with high-risk GISTs ${ }^{6}$.

Bone metastases arising from GISTs have been reported, but their true prevalence is unknown. Jati et al. ${ }^{8)}$ reported that $6(3.2 \%), 4$ and 2 of 190 GISTs patients had bone metastases, multiple bone metastases, and a solitary metastasis, respectively. The metastases involved the pelvic bone $(n=4)$, ribs $(n=4)$, vertebrae $(n=4)$, and femur $(n=3)$. The spine metastases were hypointense on T1-weighted images (WI) and hyperintense on T2WI on MRI finding. All lesions were well-marginated and osteolytic. Except 1 patient, all lesions were discrete. There were no sclerotic components in any lesion. The metastases caused cortical disruption, which led to fractures in 2 patients. There was an associated soft tissue component in 3 patients who underwent PET. The bone metastases showed intense fluorode- 
oxyglucose uptake (mean SUVmax, 8.7; range, 6.7-11) which was similar to that of liver metastases (mean SUVmax, 13.4; range, 10-20).

Di Scioscio et al. ${ }^{5}$ reported 3 GIST cases with bone metastasis and two of them showed bone and liver metastasis at the time of disease presentation. The first patient had multiple osteolytic lesions in the spine and rib. The second and third patient had the lesion in the sacrum and pelvic bone. Imatinib and zoledronic acid were given to all patients. One of them received conventional radiation therapy (cRTx). Aktan et al. ${ }^{1)}$ also reported the results of cRTx in 2 cases of GIST with spine metastasis. Palliative radiotherapy was performed at a fraction of $3 \mathrm{~Gy}$ with a total dose of $30 \mathrm{~Gy}$ on the spinal metastasis, and pain complaint significantly decreased after the radiotherapy.

Some of the surgical treatments of spinal metastasis in GISTs have been introduced. Waterman et al. ${ }^{16)}$ reported the experience of decompression and multi-level fixation surgery in metastatic lesion at T-L spine junction with acute spinal cord compression due to aggressive regional spread. Before the surgery was performed, the origin of mass was an unknown primary malignancy lesion, and histologic results confirmed that it was GIST with spinal metastases. Slimack et al. ${ }^{12)}$ also reported good surgical results in a huge metastatic lesion with spinal cord compression from GIST in the thoracic spine.

In our cases, before the surgery was performed, the patients were unable to walk due to severe radiating pain. However, after surgery was performed, the patients felt less radiating pain and were able to make ambulation. There are no complications with surgery. A surgical approach can be a treatment option in patients with spine metastasis for decompression or tumor resection. Radiosurgery or radiation therapy can be used in patients with spine metastases for palliative reasons ${ }^{14)}$. In case 2 , prior to radiosurgery, the patient had a severe back pain, but after the radiosurgery, the patient had shown that the back pain decreased.

\section{CONCLUSION}

We experienced two cases of spinal metastasis from GISTs and reviewed the literature. Spinal metastases from GISTs are rare, but they may become more prevalent due to increased patient life expectancy as well as the improvement in imaging techniques. GISTs should be considered in the workup of a patient with bone metastases with unknown primary malignancy. Aggressive surgical treatment is needed in metastatic lesions that cause spinal cord compression. However, SRS may be a good therapeutic option for unresectable severe metastatic lesions or patients with severe symptoms.

\section{CONFLICT OF INTEREST}

No potential conflict of interest relevant to this article was reported.

\section{REFERENCES}

1. Aktan M, Koc M, Yavuz BB, Kanyilmaz G: Two cases of gastrointestinal stromal tumor of the small intestine with liver and bone metastasis. Ann Transl Med 3:259, 2015

2. Amalesh T: Gastrointestinal stromal tumours (Br J Surg 2003; 90:1178-1186). Br J Surg 91:252-253, 2004

3. Bashir U, Qureshi A, Khan HA, Uddin N: Gastrointestinal stromal tumor with skeletal muscle, adrenal and cardiac metastases: an unusual occurrence. Indian J Pathol Microbiol 54:362-364, 2011

4. DeMatteo RP, Lewis JJ, Leung D, Mudan SS, Woodruff JM, Brennan MF: Two hundred gastrointestinal stromal tumors: recurrence patterns and prognostic factors for survival. Ann Surg 231:51-58, 2000

5. Di Scioscio V, Greco L, Pallotti MC, Pantaleo MA, Maleddu A, Nannini M, et al.: Three cases of bone metastases in patients with gastrointestinal stromal tumors. Rare Tumors 3:e17, 2011

6. Eisenberg BL: The SSG XVIII/AIO trial: results change the current adjuvant treatment recommendations for gastrointestinal stromal tumors. Am J Clin Oncol 36:89-90, 2013

7. Gong L, Li YH, Zhao HD, Zhao JY, Zhang W: The clinicopathologic observation, c-KIT gene mutation and clonal status of gastrointestinal stromal tumor in the sacrum. BMC Gastroenterol 9:43, 2009

8. Jati A, Tatli S, Morgan JA, Glickman JN, Demetri GD, Van den Abbele A, et al.: Imaging features of bone metastases in patients with gastrointestinal stromal tumors. Diagn Interv Radiol 18:391-396, 2012

9. Miettinen M, El-Rifai W, L HLS, Lasota J: Evaluation of malignancy and prognosis of gastrointestinal stromal tumors: a review. Hum Pathol 33:478-483, 2002

10. Murakami M, Ebisui C, Okada K, Okamura S, Murata K, Nakagomi $\mathrm{N}$, et al.: A case of liver and bone metastasis from gastrointestinal stromal tumor treated using imatinib. Gan To Kagaku Ryoho 41:99-101, 2014

11. Rauf F, Bhurgri Y, Pervez S: Gastrointestinal stromal tumors: a demographic, morphologic and immunohistochemical study. Indian J Gastroenterol 26:214-216, 2007

12. Slimack NP, Liu JC, Koski T, McClendon J, Jr., O'Shaughnessy BA: Metastatic gastrointestinal stromal tumor to the thoracic and lumbar spine: first reported case and surgical treatment. Spine J 12:e7-e12, 2012

13. Stamatakos M, Douzinas E, Stefanaki C, Safioleas P, Polyzou E, Levidou G, et al.: Gastrointestinal stromal tumor. World J Surg Oncol 7:61, 2009

14. Tezcan Y, Koç M: Gastrointestinal stromal tumor of the rectum with bone and liver metastasis: a case study. Med Oncol 28 Suppl 1:S204-S206, 2011

15. Wang ZQ, Wang S, Ye YJ, Kang YL, Sun KK, Zheng HF: Gastrointestinal mesenchymal tumors: a clinical pathologic and immunohistochemical study of 210 cases. Zhonghua Wei Chang Wai Ke Za Zhi 10:11-16, 2007

16. Waterman BR, Kusnezov N, Dunn JC, Hakim MN: Aggressive gastrointestinal stromal tumor with spinal metastases: a case report. Mil Med 180:e618-e621, 2015 